\title{
Flux Tubes in Weyl Gravity
}

\author{
V. Dzhunushaliev* \\ Institut für Mathematik, Universität Potsdam PF 601553, D-14415 Potsdam, Germany
}

\author{
H.-J. Schmidt ${ }^{\dagger}$ \\ Institut für Theoretische Physik, Freie Universität Berlin \\ and \\ Institut für Mathematik, Universität Potsdam PF 601553, D-14415 Potsdam, Germany
}

\begin{abstract}
The spherically symmetric solutions in Weyl gravity interacting with $U(1)$ or $S U(2)$ gauge fields are examined. It is shown that these solutions are conformally equivalent to an infinite flux tube with constant (color) electric and magnetic fields. This allows us to say that Weyl gravity has in some sense a classical confinement mechanism. We discuss a possible connection with flux tubes in quantum chromodynamics.
\end{abstract}

Typeset using REVTEX

\footnotetext{
*E-Mail Addresses : dzhun@rz.uni-potsdam.de and dzhun@freenet.bishkek.su; permanent address: Dept. Theor. Phys., Kyrgyz State National University, Bishkek 720024, Kyrgyzstan

${ }^{\dagger}$ http://www.physik.fu-berlin.de/ ${ }^{\sim}$ hjschmi hjschmi@rz.uni-potsdam.de
} 


\section{INTRODUCTION}

The conformal Weyl gravity has a long history. Initially it was introduced as an attempt

of generalizing Einstein gravity to obtain a common description for the unified theory of gravity+electromagnetism, see [1]. This attempt was unsuccessful, however, now this theory attracts much attention of gravity researchers, see [2] and the references cited there.

In this paper we want to demonstrate an unexpected property of this theory of gravity as a classical confinement for $U(1)$ and $S U(2)$ gauge fields.

What is it a confinement in QCD? There is an assumption that the force lines of colorelectrical field between quark and antiquark are confined to a flux tube stretched between these particles. This object is a complete quantum phenomenon. The difficulties to explaining this tube is that the $S U(3)$ nonabelian gauge field is a strongly nonlinear field, and we cannot use the Feynman diagramm technique.

In this paper we will show that spherically symmetric solutions in Weyl gravity model in the some sense properties of the classical confinement mechanism.

\section{II. (2+2)-DECOMPOSITION OF THE METRIC}

Here we examine the static spherically symmetric solutions in four-dimensional (4D) Weyl gravity with $U(1)$ and $S U(2)$ gauge fields. The most general spherically symmetric metric takes the form:

$$
d s^{2}=g_{A B} d x^{A} d x^{B}-a^{2}(r) d \Omega^{2},
$$

where $g_{A B}$ depends on the coordinates $x^{A}$ only, has curvature scalar $X$ and signature $(+-)$, ( $A, B=0,1, x^{0}=t, x^{1}=r$, but $r$ need not be space-like) and $d \Omega^{2}=d \theta^{2}+\sin ^{2} \theta d \varphi^{2}$ is the metric on the unit $S^{2}$ sphere. As the Weyl gravity, Maxwell electrodynamic and Yang-Mills theory are conformally invariant theories we can work with the following metric which is conformally equivalent to (1) metric:

$$
d s^{2}=\tilde{g}_{A B} d x^{A} d x^{B}-d \Omega^{2}
$$

where $\tilde{g}_{A B}=g_{A B} / a^{2}$. The hypersurface $t=$ const for this metric is $R \times S^{2}$, i.e. a tube with the constant cross section as the $S^{2}$ sphere.

Now we repeat a $(2+2)$-decomposition technique following to paper [2]. 
Let the 4D metric be

$$
d s^{2}=g_{i j} d x^{i} d x^{j}, \quad i, j=0, \ldots 3
$$

Let we have the following ansatz:

$$
d s^{2}=d \sigma^{2}-d \tau^{2}
$$

where $d \sigma^{2}$ and $d \tau^{2}$ are both 2-dimensional. The metric

$$
d \sigma^{2}=\tilde{g}_{A B} d x^{A} d x^{B}, \quad A, B=0,1 .
$$

The other 2-dimensional metric

$$
d \tau^{2}=g_{\alpha \beta} d x^{\alpha} d x^{\beta}, \quad \alpha, \beta=2,3,
$$

where $g_{\alpha \beta}$ depends on the $x^{\alpha}$ only, has curvature scalar $Y$ and signature $(++)$. .

The lagrangian for the conformal Weyl gravity interacting with the gauge field is:

$$
\mathcal{L}=-C_{i j k l} C^{i j k l}-\frac{\kappa}{4} \operatorname{tr}\left(F_{l m} F^{l m}\right),
$$

here $C_{i j k l}$ is the conformally invariant Weyl tensor, $F_{l m}$ is the tensor of field strength for the gauge potential and we keep both possible signs of $\kappa$. The Bach equations are:

$$
B_{i j}=\kappa T_{i j}
$$

where

$$
\begin{array}{r}
B_{i j}=B_{i j}^{(1)}+B_{i j}^{(2)} \\
B_{i j}^{(1)}=-\square R_{i j}+2 R_{i ; j k}^{k}-\frac{2}{3} R_{; i j}+\frac{1}{6} g_{i j} \square R \\
B_{i j}^{(2)}=\frac{2}{3} R R_{i j}-2 R_{i k} R_{j}^{k}-\frac{1}{6} R^{2} g_{i j}+\frac{1}{2} g_{i j} R_{k l} R^{k l} .
\end{array}
$$

It can be shown that for our (3), (5), (6) ansatz:

$$
\begin{gathered}
B_{A B}=\frac{1}{3} X_{; A B}+g_{A B}\left(\frac{1}{6} \square Y-\frac{1}{3} \square X+\frac{1}{12} Y^{2}-\frac{1}{12} X^{2}\right)=\kappa T_{A B}, \\
B_{\alpha \beta}=\frac{1}{3} Y_{; \alpha \beta}+g_{\alpha \beta}\left(\frac{1}{6} \square X-\frac{1}{3} \square Y+\frac{1}{12} X^{2}-\frac{1}{12} Y^{2}\right)=\kappa T_{\alpha \beta} .
\end{gathered}
$$

\footnotetext{
${ }^{1}$ For us is only important that $d \tau^{2}=d \theta^{2}+\sin ^{2} \theta d \varphi^{2}$ with $Y=2$.
} 


\section{III. $U(1)$ FLUX TUBE}

In Ref. [3] the static, spherically symmetric solution for the Bach-Maxwell equations is given:

$$
d s^{2}=\left(\frac{r^{2}}{a_{0}}+b r+c+\frac{d}{r}\right) d t^{2}-\frac{d r^{2}}{\left(\frac{r^{2}}{a_{0}}+b r+c+\frac{d}{r}\right)}-r^{2} d \Omega^{2},
$$

with an electromagnetic one-form potential:

$$
A=A_{i} d x^{i}=A_{t} d t
$$

where $a_{0}, b, c, d$ and $q$ are some constants with the following relation (16) where $q$ is the electric charge and the parameter $\beta$ used in [3] is related to our conventions via $\beta \kappa=4$ :

$$
3 b d-c^{2}+1+\frac{3}{8} q^{2} \kappa=0
$$

If $b=c=d=0$ we have the following solution:

$$
d s^{2}=r^{2}\left(\frac{d t^{2}}{a_{0}}-\frac{a_{0}}{r^{4}} d r^{2}-d \Omega^{2}\right) .
$$

We can introduce new dimensionless coordinates $t^{\prime}=t / a_{0}$ and $x=\sqrt{a_{0}} / r$ then:

$$
d s^{2}=\frac{a_{0}}{x^{2}}\left(d t^{2}-d x^{2}-d \Omega^{2}\right)
$$

with $x \in(-\infty,+\infty)$ and we rename $t^{\prime} \rightarrow t$. This metric is conformally equivalent to

$$
d s^{2}=d t^{2}-d x^{2}-d \Omega^{2}
$$

which represents the cartesian product of a flat and a non-flat 2-space of constant curvature. (For ease of comparison we mention the following result from [1]: The cartesian product of two 2-spaces of constant curvature solves the Bach equation if and only if $X^{2}=Y^{2}$.)

Because of the conformal invariance of the Weyl-Maxwell theory we can work in this paragraph with this metric (19). We already mentioned that this is a tube and now we can say that this tube is filled by an electric field $E_{1}=F_{01}=q, \quad F_{02}=F_{03}=0.9$

Now we wish to obtain this solution including a magnetic field and using the (2+2)decomposition technique. It can seen that ansatz (15) can be generalized by introducing the monopole, where $Q$ is the magnetic charge, $F_{23}=Q, \quad F_{12}=F_{13}=0$ :

\footnotetext{
${ }^{2}$ Remember that we use a dimensionless coordinate system.
} 


$$
A=\omega(r) d t-Q \cos \theta d \varphi .
$$

The Bach-Maxwell equation for metric (19) looks as:

$$
Y^{2}-X^{2}=6 \kappa\left(Q^{2}+q^{2}\right),
$$

here $X=0$ and $Y=2$ for the unit $S^{2}$-sphere. Note: As one knows, a Lorentz boost in the $x-t$-plane mixes electric and magnetic fields, but the term $Q^{2}+q^{2}$ remains invariant. The Maxwell equations give us:

$$
\omega=q x+\omega_{0},
$$

and finally

$$
\kappa\left(Q^{2}+q^{2}\right)=\frac{2}{3} .
$$

The electric and magnetic fields are:

$$
\begin{array}{r}
E_{x}=F_{t x}=q, \\
H_{x}=\epsilon_{x \theta \varphi} \sqrt{-g} F^{\theta \varphi}=Q .
\end{array}
$$

here $\epsilon_{i j k}$ is the totally antisymmetric Levi-Civita tensor of the spatial metric. Thus, this (19), (22) and (23) solution of Bach-Maxwell equations with the constant electric and magnetic fields across the area $4 \pi$ of $S^{2}$ sphere we can name as a flux tube. The fluxes of electric and magnetic fields across unit area of $S^{2}$ sphere are:

$$
\begin{aligned}
\Phi_{E} & =4 \pi E_{x}=4 \pi q, \\
\Phi_{H} & =4 \pi H_{x}=4 \pi Q .
\end{aligned}
$$

This is analogous to the Levi-Civita-Robertson-Bertotti solution in Einstein gravity [ [4], [5], [6].

Finally, we would like to pay attention to an exceptional simplicity of (19), (24), (25) solution with the constant electrical, magnetic fields and metric components.

\section{IV. $S U(2)$ FLUX TUBE}

The spherically symmetric ansatz for an $S U(2)$ gauge field is a monopole-like potential written in a polar coordinate system: 


$$
\begin{aligned}
& A_{t}^{a}=v(r)\{\cos \theta ; \sin \theta \sin \varphi ; \sin \theta \cos \varphi\} \\
& A_{r}^{a}=0, \\
& A_{\theta}^{a}=(1-f(r))\{0 ; \cos \varphi ;-\sin \varphi\} \\
& A_{\varphi}^{a}=(1-f(r)) \sin \theta\{\sin \theta ;-\cos \theta \sin \varphi ;-\cos \theta \cos \varphi\}
\end{aligned}
$$

The Yang-Mills equations for the metric

$$
d s^{2}=e^{2 \nu} d t^{2}-d r^{2}-d \Omega^{2}
$$

are:

$$
\begin{array}{r}
f^{\prime \prime}-f^{\prime} \nu^{\prime}-f\left(f^{2}-1\right)+e^{-2 \nu} f v^{2}=0 \\
v^{\prime \prime}-v^{\prime} \nu^{\prime}-2 v f^{2}=0
\end{array}
$$

and the Bach equations are:

$$
\begin{aligned}
\frac{1}{3} X_{; B}^{; A}+\delta_{B}^{A}\left(-\frac{1}{3} \square X+\frac{1}{12} Y^{2}-\frac{1}{12} X^{2}\right) & =\kappa T_{B}^{A}, \\
\delta_{\beta}^{\alpha}\left(\frac{1}{6} \square X+\frac{1}{12} X^{2}-\frac{1}{12} Y^{2}\right) & =\kappa T_{\beta}^{\alpha} .
\end{aligned}
$$

here $Y=2$ is the Ricci scalar of the sphere $S^{2}$. The energy-momentum tensor for this $S U(2)$ gauge potential is:

$$
\begin{aligned}
T_{t}^{t} & =f^{\prime 2}+\frac{1}{2} e^{-2 \nu} v^{\prime 2}+\frac{1}{2}\left(f^{2}-1\right)^{2}+e^{-2 \nu} f^{2} v^{2}, \\
T_{r}^{r} & =-f^{\prime 2}+\frac{1}{2} e^{-2 \nu} v^{\prime 2}+\frac{1}{2}\left(f^{2}-1\right)^{2}-e^{-2 \nu} f^{2} v^{2} \\
T_{\theta}^{\theta}=T_{\varphi}^{\varphi} & =-\frac{1}{2} e^{-2 \nu} v^{\prime 2}-\frac{1}{2}\left(f^{2}-1\right)^{2}
\end{aligned}
$$

The traces of eqs. (35) and (36) and the remainder of the $\left(\begin{array}{l}t \\ t\end{array}\right)$ equation give us:

$$
\begin{gathered}
X_{; t}^{; t}-X_{; r}^{; r}=6 \kappa\left(f^{\prime 2}+e^{-2 \nu} f^{2} v^{2}\right), \\
\square X+\frac{1}{2}\left(X^{2}-Y^{2}\right)=-3 \kappa\left[e^{-2 \nu} v^{\prime 2}+\left(f^{2}-1\right)^{2}\right] .
\end{gathered}
$$

We see that in this case these Bach-Yang-Mills equations are differential equations of $4^{\text {th }}$ order. Only one way is that exclude the terms with $e^{-2 \nu}$ in (40) and (41) equations: it is necessary that $f=0$ for (40) and $e^{-2 \nu} v^{\prime 2}=$ const for (41). It is easy to show that Yang-Mills equation (33) and (34) have such solution: 


$$
\begin{gathered}
f=0, \\
v^{\prime}=q e^{\nu}
\end{gathered}
$$

here $q$ is the some constant (color electric charge). In this case the simplest solution of (33), (34) (40), (41) is

$$
\begin{aligned}
X & =0, \\
Y & =2, \quad \text { the curvature of } S^{2}, \\
Y^{2} & =6 \kappa\left(q^{2}+1\right) \\
v & =q x+v_{0}
\end{aligned}
$$

here $v_{0}$ is the some constant.

\section{PHYSICAL DISCUSSION}

It can be shown that the $S U(2)$ solution (42), (43) is in fact the $U(1)$ solution. To see this most directly one can apply the following gauge transformation to the potentials of Eqs. (28 - 31) and taking in account (42) and (47)

$$
A_{\mu}^{\prime}=S^{-1} A_{\mu} S-i\left(\partial_{\mu} S^{-1}\right) S
$$

where

$$
S=\left(\begin{array}{cc}
\cos \frac{\theta}{2} & -e^{-i \varphi} \sin \frac{\theta}{2} \\
e^{i \varphi} \sin \frac{\theta}{2} & \cos \frac{\theta}{2}
\end{array}\right)
$$

with this gauge transformation we find that the gauge potentials become

$$
\begin{aligned}
& A_{\theta}^{\prime a}=(0 ; 0 ; 0), \\
& A_{\varphi}^{\prime a}=(\cos \theta-1)(0 ; 0 ; 1), \\
& A_{t}^{\prime a}=v(r)(0 ; 0 ; 1),
\end{aligned}
$$

so that only the $\sigma^{3}$ direction in isospin space is non-zero. The calculation of the magnetic and electric fields is now the same as for the $U(1)$ case since the "Abelian" gauge transformation given by Eqs. (48) - (49) brings us to a gauge where the non-Abelian fields take on an Abelian form. 
This means that in the $S U(2)$ flux tube solution happens the dynamical symmetry breakdown from $S U(2)$ gauge group to $U(1)$ gauge group: $S U(2) \rightarrow U(1)$. Note: The same arguments apply also to the case that we replace $S U(2)$ by $S U(n)$ for any $n>2$.

Another remarkable peculiarity for both these solutions is that they are the flux tubes with the constant flux of (color) electric and/or (color) magnetic fields across the cross section $S^{2}$ of space-like hypersurface $t=$ const.

We would like to compare these gravitational flux tube (GFT) with the flux tube in QCD. In QCD there is an assumption that the force lines of the $S U(3)$ gauge field between quarkantiquark pair are confined into a tube in the consequence of strong interaction of nonabelian chromodynamic field. We want to underline the same analogy between these two objects in the QCD and the Weyl conformal gravity. Of course this is not an exact correspondence as the GFT is whole spacetime but the flux tube in QCD exists in some external space but we repeat again that we say about some approximate correlation between these so remote theories. It is surprising in general that there is some kind of correlation between the objects in classical gravity and in quantum nonabelian field theory. It has to be mentioned that now there is a $A d S^{5} \times S^{5}$ correspondence [7], [8] and [9], but for this correspondence the existence of superspace is important.

Finally we want to extract the following physical pecularities of the obtained flux tube solutions in conformal Weyl gravity:

- In Weyl gravity there are the GFT solutions.

- In $S U(2)$ GFT takes place the dynamical breakdown of gauge symmetry: $S U(2) \rightarrow$ $U(1)$.

- Flux tube solutions in Weyl gravity can be interpreted as some indication that this gravity theory has a classical confinement mechanism in the following sense: two opposite electrical and/or magnetic charges carried at infinite distance form the infinite flux tube space filled by constant electric and/or magnetic fields. We underline once again that this confinement mechanism is a classical and not a quantum effect.

- There is an analogy between some objects in classical gravity and quantum nonabelian field theory. Is this analogy accidental or is there some deeper connection between classical gravity and quantum nonabelian field theory? 


\section{ACKNOWLEDGEMENT}

Financial support from DFG and A.-v.-Humboldt-Found. is gratefully acknowledged. 


\section{REFERENCES}

[1] H. Weyl, Sitzungsber. Preuss. Akad. d. Wiss. Berlin, Phys.-Math. Kl. 465 (1918).

[2] V. Dzhunushaliev and H.-J. Schmidt, gr-qc/9908049 (1999).

[3] R. J. Riegert, Phys. Rev. Lett. 53, 315 (1984).

[4] T. Levi-Civita, Rend. Atti acad. Naz. Lincei 26, 529 (1917).

[5] I. Robinson, Bull. Akad. Pol. 7, 351 (1959).

[6] B. Bertotti, Phys. Rev. 116, 116 (1959).

[7] G. 't Hooft, gr-qc/9310026 (1993).

[8] L. Susskind, J. Math. Phys. 36, 6377 (1995).

[9] C. Thorn, Published in Sakharov Conf. on Physics, Moscow 447 (1991); hep-th/9405069. 